\title{
The Evolution of Very Early Onset Inflammatory Bowel Disease, Autoimmune Hepatitis, and Primary Sclerosing Cholangitis in a Young Girl
}

\author{
Aditi Dey ${ }^{a}$ Jacquelin Peck ${ }^{a} \quad$ Michael Wilsey ${ }^{a}$ b Jolan Walter ${ }^{c}$ \\ Johnny Nguyen ${ }^{d} \quad$ Ignacio Gonzalez-Gomez ${ }^{d} \quad$ Sara Karjoo ${ }^{a}$ b, e \\ aDepartment of Pediatrics, Johns Hopkins All Children's Hospital, St. Petersburg, FL, USA; \\ ${ }^{b}$ Department of Pediatrics, University of South Florida Morsani College of Medicine, Tampa, \\ FL, USA; 'Department of Internal Medicine, Allergy and Immunology, University of South \\ Florida Morsani College of Medicine, Tampa, FL, USA; ${ }^{d}$ Department of Pathology, Johns \\ Hopkins All Children's Hospital, St. Petersburg, FL, USA; eDepartment of Clinical Sciences, \\ Florida State University, Tallahassee, FL, USA
}

Keywords

Autoimmune hepatitis · Crohn's disease - Very early onset inflammatory bowel disease ·

Primary sclerosing cholangitis

\begin{abstract}
Very early onset inflammatory bowel disease, autoimmune hepatitis (AIH), or primary sclerosing cholangitis (PSC) alone is a rare condition in young children. The combination of all 3 autoimmune disorders in a 16-month-old child is even rarer. The onset and etiology of these diseases is multifactorial and typically unknown. However, when the children are diagnosed, the accepted view point is that the inflammation was likely present for months to years prior. This case is unique because the gastrointestinal problems started from infancy, and evolved to the development of Crohn's disease, AlH, and PSC at a very early age. This case helps bring to light that very early onset autoimmune disorders may in fact present with symptoms of feeding difficulties, growth failure, and formula intolerance. Patients may be diagnosed initially with allergic enterocolitis in infancy. Although few children with these symptoms evolve to develop autoimmune diseases at an older age, clinicians should consider following these children more closely. This case also demonstrates how hard it is to diagnose very early onset autoimmune disorders, as they mimic other illnesses.
\end{abstract}


Dey et al.: Young Girl with VEO-IBD, AIH, and PSC

\section{Introduction}

Very early onset inflammatory bowel disease (VEO-IBD) occurs in children under the age of 6 years and infantile onset of inflammatory bowel disease (IBD) occurs under the age of 2 years [1,2]. The incidence of pediatric IBD has been increasing [1, 3]. Other conditions such as cow milk protein allergy, primary immune deficiency, infections, and eosinophilic gastroenteritis have similar clinical presentations and should be considered when evaluating for early onset IBD [1]. Possible mechanisms of development of VEO-IBD include excess antibiotic use, decreased exposure to parasites or infections, and dietary additions of emulsifiers and surfactants that all alter the gut microbiota [3]. Autoimmune hepatitis (AIH) can occur at any age-group or gender, but predominantly presents in adolescent females [4]. This disease is rare in early childhood. However, cases of AIH have been reported in infancy [5]. There are 2 types of AIH. Type 1 is characterized by the presence of antinuclear antibodies (ANA) and/or anti-smooth muscle antibodies (ASMA), and type 2 is characterized by anti-liver-kidney-microsomal antibody or liver cytosol type 1 antibody [4]. Primary sclerosing cholangitis (PSC) can be associated with IBD and AIH [6]. There have been case reports of neonatal sclerosing cholangitis as young as 1 month of age, but generally, PSC is very rare in young children as well [6]. In addition, AIH-PSC overlap has been described predominately in females of younger ages [6]. The average age of diagnosis of AIH and PSC/ overlap syndrome has been reported to be 10.41 years [5]. These patients with overlap have elevated serum immunoglobulin levels, positive ANA, or ASMA [6]. Disordered microbiota is suspected to be the link among IBD, AIH, and PSC. Pro-inflammatory bacterial-derived peptides and secondary bile acids with repeated episodes of low-grade bacterial infections are suspected to lead to hepatobiliary disease in PSC [6, 7]. Lymphocytes are then activated to recognize the bowel and hepatic endothelium with memory T-cells that induce inflammation [6]. Vancomycin is used to treat IBD and PSC, due to its effects as an antimicrobial and an immunomodulator [8]. What is unique about this case is that the patient developed Crohn's disease, AIH, and PSC around the age of 1 year. Initially, this young girl presented as an infant with poor growth, reflux, feeding difficulties, and suspected allergic enterocolitis. The diagnosis of VEO-IBD, AIH, and PSC was highly considered when she was a toddler. However, when she turned 6 years of age, her disease progressed, and the diagnosis of Crohn's disease, AIH, and PSC was unmistakable.

\section{Case Report}

A 2-month-old girl child with a history of eczema initially presented for reflux, colic, feeding difficulties, and failure to thrive. The patient's maternal family history was significant for Crohn's and ulcerative colitis, but her mother was healthy. The patient's father and his family did not have a history of autoimmune disease. The physical exam upon presentation was normal, except for well-controlled eczema. She had several formula changes due to feeding difficulties and was subsequently diagnosed with reflux and cow milk protein enterocolitis. She had a normal upper gastrointestinal tract radiography and oral pharyngeal motility swallow study, and she was followed by feeding therapy. She was treated with a proton pump inhibitor and was started on high-calorie hydrolyzed formula to support with weight gain until 1 year of age. At 1 year of age, dairy was added to the diet. Diarrhea started at 14 months of age. By 16 months of age, diarrhea progressed to include blood. Stool study results included negative stool culture, positive fecal occult blood, and significantly elevated calprotectin (shown in Table 1). An upper and lower endoscopy at the age of 16 months showed mild chronic active gastritis, and chronic active colitis with eosinophils, cryptitis, crypt abscess

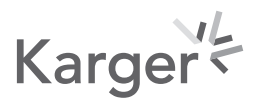


Table 1. Calprotectin trends over the patient's ages 16 months- 6 years

\begin{tabular}{ll}
\hline Age & Calprotectin, $\mu \mathrm{g} / \mathrm{g}$ \\
\hline 16 months & $>2,000$ \\
20 months & $>2,000$ \\
21 months & 317.8 \\
24 months & 671.4 \\
6 years & 2,340 \\
\hline
\end{tabular}

Table 2. Pathology reports for procedures performed at 16 months and at 6 years of age

\begin{tabular}{ll}
\hline 16 months & \\
Duodenum & Intact duodenal mucosa without inflammation or microorganisms \\
Stomach, antrum & Mild chronic active gastritis. No granulomas or microorganism \\
Esophagus & Intact esophageal mucosa without inflammation or microorganisms \\
Colon, sigmoid segment & Chronic active colitis with cryptitis. Crypt abscess formation, \\
& granulomas or microorganisms not identified \\
Comment & The morphologic features seen in colonic biopsies are nonspecific and \\
& may be seen in conditions as variable as bowel preparation, allergic \\
(dietary protein) colitis, infection and in this patient's age-group, & rarely, IBD \\
years & Mild chronic duodenitis with focal active cryptitis and crypt \\
Duodenum & alteration/hyperplasia. No evidence of granulomas or microorganisms \\
Stomach, antrum & Mild chronic gastritis with focal active cryptitis. Negative for \\
helicobacter microorganisms & Esophageal mucosa, no diagnostic pathologic abnormalities \\
Esophagus & Mild chronic ileitis with focal active cryptitis and focal crypt alteration. \\
Small intestine, terminal ileum & No evidence of granulomas, crypt abscess, or microorganisms \\
Colon, cecum to rectum & $\begin{array}{l}\text { Mild chronic colitis with focal active cryptitis and crypt alteration. No } \\
\text { evidence of granulomas, crypt abscess, or microorganisms } \\
\text { Comment }\end{array}$ \\
\hline
\end{tabular}

IBD, inflammatory bowel disease.

formation, without granulomas (shown in Table 2; Fig. 1). The histology showed nonspecific morphologic features that may be seen in various conditions such as allergic colitis, infection, and IBD. The medical team decided that these were features of an allergic enterocolitis. She was switched to amino acid-based formula at 17 months of age and asked to avoid dairy. Despite months on this formula, she continued to have diarrhea and intermittent hematochezia. She was transitioned to $100 \%$ amino acid-based formula at $40 \mathrm{cal} / \mathrm{oz}$, given both orally and via a nasogastric tube to mimic enteral nutritional therapy treatment in case this is early Crohn's disease. Eventually, the patient required a surgical gastrostomy tube for persistent feeding issues. A second calprotectin at 20 months of age did not show improvement (shown in Table 1). At the age of 20 months, a stool Clostridioides difficile PCR was positive and was performed due to chronic intermittent hematochezia. Although Clostridioides difficile can be considered normal flora in children under 2-3 years, the patient's symptoms of recurrent hematochezia warranted expanded evaluation. Treatment 
Fig. 1. Initial biopsy at 16 months. Colonic mucosa shows mild glandular architecture distortion and regenerative changes with hypercellularity and decreased mucin in goblet cells. The lamina propria is expanded with an increased number of lymphocytes, plasma cells, eosinophils, and scattered neutrophils. Significant eosinophilis noted in the lamina propria (white arrow). Focal scattered cryptitis is present (blue arrows). H\&E. $\times 100$ and $\times 300$ nominal magnifications.

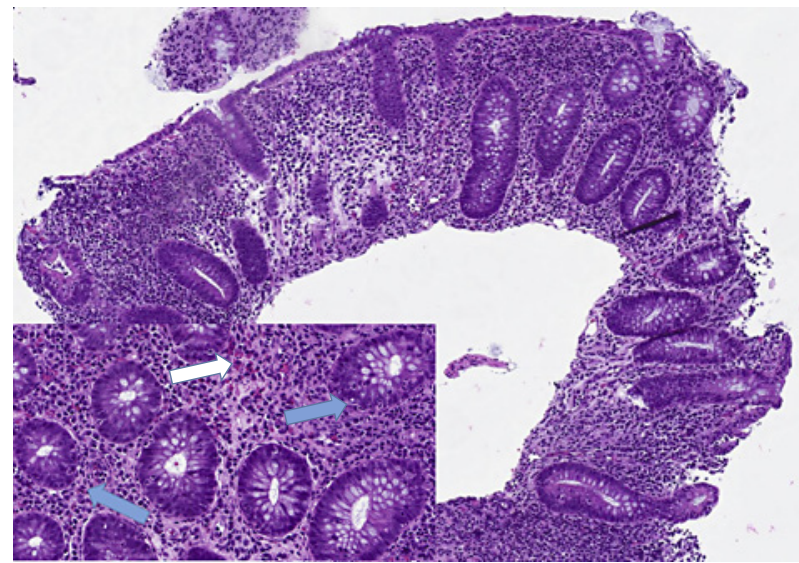

included multiple courses of metronidazole and a 1-month vancomycin taper that was monitored by the infectious disease specialist. Fecal transplant from a healthy mother was performed at 27 months of age via a nasogastric tube for the treatment of recurrent Clostridioides difficile. Hematochezia resolved after transplant. However, 2 weeks post-transplant, she developed a 1-month fever of unknown origin, requiring hospital admission. Fever workup revealed elevated liver enzymes (alanine amino transferase $86 \mathrm{IU} / \mathrm{L}$, aspartate amino transferase $105 \mathrm{IU} / \mathrm{L}$, and gamma glutamyl transferase $80 \mathrm{U} / \mathrm{L}$, with normal total bilirubin $0.4 \mathrm{mg} / \mathrm{dL}$, albumin $4.2 \mathrm{~g} / \mathrm{dL}$, alkaline phosphatase $209 \mathrm{IU} / \mathrm{L}$ ), a positive ANA (1:640), and ASMA (1:40). Liver biopsy (shown in Fig. 2a-c) confirmed a diagnosis of AIH-PSC overlap syndrome. The magnetic resonance cholangiopancreatography did not show any large bile duct abnormalities or strictures. The Meckel scan was normal. A prior baseline hepatic function panel before the admission to the hospital was not performed/available for comparison. The patient was referred to immunology for concern for immunodeficiency. She had normal white blood cell counts $(11.910 \mathrm{e} 3 / \mu \mathrm{L})$, lymphocyte subsets, and normal immunoglobulin levels $(1,050 \mathrm{mg} / \mathrm{dL})$. Extensive genetic testing and chromosomal microarray were unremarkable. A 207-gene panel linked to primary antibody deficiencies revealed heterozygous variants of unknown significance for LYST and PRKDC genes without changes in copy number variation. Both genes are linked to autosomal recessive immune disorders, and the significance of these mutations is unknown.

With her liver findings and intermittent hematochezia, she was now suspected to have Crohn's disease, AIH, and PSC, and treatment was initiated. Around this time, a secondary consultation was sought at a well-respected children's hospital's IBD center, where specialists agreed with the diagnosis of VEO-IBD, AIH, and PSC. The patient completed a 1.5-month course of prednisone for the treatment of AIH which improved liver enzymes (alanine amino transferase $38 \mathrm{IU} / \mathrm{L}$, aspartate amino transferase $20 \mathrm{IU} / \mathrm{L}$, and gamma glutamyl transferase $35 \mathrm{U} / \mathrm{L}$, with normal total bilirubin $0.3 \mathrm{mg} / \mathrm{dL}$, albumin $3.3 \mathrm{~g} / \mathrm{dL}$, and alkaline phosphatase $190 \mathrm{IU} / \mathrm{L}$ ). She was also concurrently on ursodeoxycholic acid and vancomycin for the treatment of PSC. Sulfasalazine was also prescribed to help with her bowel disease. The patient's rectal bleeding briefly improved, and her stool consistency and calprotectin improved (shown in Table 1).

At 35 months of age she was started on azathioprine and sulfasalazine, to try to wean her off of chronic steroid treatment. The combination helped improve her liver enzymes and rectal bleeding. However, 1 month after starting the medications, she developed severe vomiting, abdominal pain, and was admitted to the hospital with acute pancreatitis (lipase $1,309 \mathrm{U} / \mathrm{L}$ ). All medications were held. When she recovered, she was started on sulfasalazine 
Fig. 2. a H\&E. $\times 200$ total magnification. There is interface lymphocytic inflammation, focally spilling over into the adjacent hepatocytes beyond the limiting plate. The inflammation contains few mature plasma cells (blue arrows), scattered histiocytes, and eosinophils (inset, red arrows). b H\&E. $\times 400$ total magnification. Focal lobular inflammation and hepatocyte injury. c H\&E. $\times 300$ total magnification. Stage 3 portal fibrosis including areas of dense concentric fibrosis surrounding bile ducts, highlighted by trichrome special staining (inset).

Fig. 3. Biopsy at 6 years. The colon shows mild distortion of glandular architecture with variable hypercellularity and decreased mucin in goblet cells. There is expansion of the lamina propria with a mixed infiltrate of lymphocytes, plasma cells, scattered eosinophils, and fewer neutrophils. Focal cryptitis is noted (blue arrows). H\&E. $\times 100$ and $\times 300$ (inset, arrows), nominal magnification.
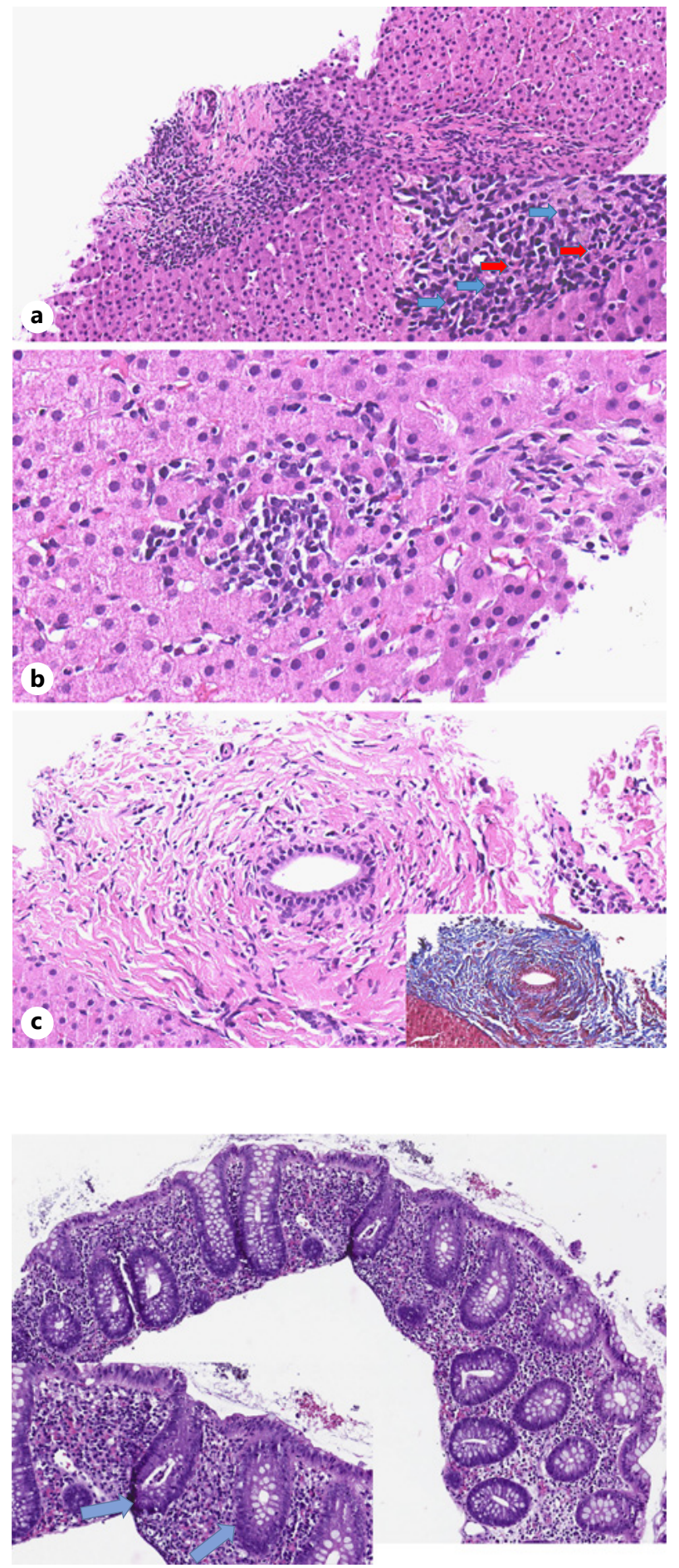

monotherapy. Due to this side effect and the patient's young age, the family did not want her to be on any other immune-modulating medications or biologic therapy. As per her mother's request, the patient was treated mainly with sulfasalazine and supportive medications. For close to 2 years, she was on and off of prednisone and budesonide in combination with sulfasalazine. She remained on 80-100\% amino acid-based nutritional therapy viagastrostomy tube, and very minimal solid food intake due to her continued oral aversions. 
However, she had a significant episode of hematochezia at the age of 6 years. She had elevated INR (1.59) and was started on oral vitamin K. This episode was complicated by the development of a nonhealing fissure, rectal bleeding, and persistent elevated liver enzymes. Repeat testing at the age of 6 years showed elevated ASMA at 1:80. Histology from upper and lower endoscopy showed inflammation now consistent with Crohn's disease (shown in Table 2; Fig. 3). The calprotectin was even higher (shown in Table 1). Discussions regarding treatment occurred and included second opinions with 3 other top children's hospitals with IBD centers. After much discussion, the patient was started on injectable anti-TNF while on prednisone. She is following endocrinology for concerns of adrenal insufficiency while weaning off steroids. She has been referred to genetics to evaluate for monogenic gene mutations typically found in VEO-IBD and for mevalonate kinase deficiency (MKD). Treatment of her liver disease with a combination immunomodulator is in consideration but has not been started until response to anti-TNF is observed.

\section{Discussion/Conclusion}

This case demonstrated the difficulty that clinicians face when diagnosing VEO-IBD, which can mimic other common illnesses in this age-group such as allergic enterocolitis. The pathologic features of VEO-IBD include eosinophils in crypts and apoptotic crypt cells [3,9], which are not typically seen in older patients with IBD. The eosinophilia seen in VEO-IBD involves the mucosa of the crypt, lamina propria, and surface epithelium [9]. The finding of eosinophilia can cause confusion with allergic or eosinophilic gastrointestinal disorders [3] as it did in this clinical case. In addition, villous blunting with or without inflammation can be seen [9]. More typical histological findings of older-onset IBD include acute inflammation like cryptitis, crypt abscesses with the presence of chronic changes like basal cell expansion, granulomas, Paneth cell metaplasia, mucin depletion, and crypt distortion, branching, and atrophy [3]. Endoscopically, patients with VEO-IBD were found to have hemorrhagic mucosa, cobblestoning with linear ulcers, duodenitis, duodenal ulcers, and predominantly colonic disease/ colitis [9]. Polygenic forms of IBD with hundreds of genetic susceptibility loci have been described in adolescent and adult onset IBD [10]. In comparison, children 5 years and younger have been reported to have monogenic mutations, including IL10RA, IL10RB, IL10 ligand and receptors, and XIAP $[10,11]$. IL-10 is an anti-inflammatory cytokine secreted by dendritic cells, natural killer cells, eosinophils, mast cells, macrophages, B-cells, and CD4+ T-cell subsets (Th2 cells, Th1 cells, Th17 cells, and Treg) [11]. These monogenic defects have been found to disrupt epithelial barrier and epithelial response [12]. These defects have been linked to reduced bacterial clearance by neutrophil granulocytes/phagocytes, hyperactivation of immune responses (due to defects in immune inhibitory mechanisms like IL-10 signaling), disruption of T- and B-cell selection and activation, and dysfunctional T-cell activity [10]. Although the patient in this clinical case did not have all the features, MKD should also be considered. MKD is an autosomal recessive inborn error of metabolism in the gene for mevalonate kinase, which results in reduced activity of the mevalonate kinase enzyme [13]. The defect causes spontaneous recurrent inflammation that clinically presents with high fever, oral ulcers, cervical lymphadenopathy, nausea, vomiting, diarrhea, skin rashes, and arthritis [13].

Furthermore, patients with IBD have intestinal microbiota differences as compared to healthy individuals [14]. There is a vulnerable window of time for the development of the intestinal microbiota between birth and 3 years of age [14]. Genetic and environmental factors (smoking, diet, air pollution, and antibiotics) can increase susceptibility to VEO-IBD [14]. Murine studies have shown interactions between microbiota and increased T-cell activation

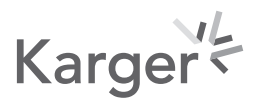


that contribute to inflammation and may increase the development of IBD [14]. The microbiota metabolites can be transferred from the mother to the child, affecting the developing immune response [14]. In intestinal dysbiosis, evidence suggests that patients with pathological microbiota demonstrate downregulation of Th2 cells $[15,16]$. This results in increased inflammation and depression of regulatory feedback mechanisms. Patients with autoimmune disorders may have dysregulation of the innate or adaptive immune system including $\mathrm{T}$ and $B$ cells [17]. There are many other areas of immune dysregulation that leads to the development of VEO-IBD. Highly activated T cells produce gamma-interferon, IL-17, and IL-22 [14]. As previously discussed, patients with VEO-IBD may have mutations in IL-10 receptor, therefore increasing IL-17 production [14]. This and the increased macrophage expression of IL-1-beta can lead to immune dysregulation [14]. The mucosal T cells can also have deficiency in branch chain glycosylation [14], which affects T-cell function. Atypical T cells have been found to interact with the gamma-delta T-cell receptor at the tip of the villi, a receptor that also changes due to interactions with the microbiota [14]. These T cells regulate the intestinal barrier and tight junction proteins [14]. Therefore, there may be an interaction among conventional T cells, unconventional T cells, the microbiota, and the epithelial surface [14] that leads to inflammation. Our patient had persistent Clostridioides difficile, and suspected immune dysregulation. Her immune and genetic workup has not identified a known immunodeficiency or disease, but testing is still ongoing. Similarly, alterations in T-cell regulation have been described in early onset AIH. The gene susceptibility for AIH is linked to the class II region of the major histocompatibility complex [18]. In children, this region has been identified to be HLA-DRB1*1301 [18]. Killer cell immunoglobulin-like receptors (KIRs) are expressed on natural killer cells and some subset of T cells [18]. A study of children with early onset type $1 \mathrm{AIH}$ showed abnormalities of KIR genes [18]. It is considered that naïve CD4+ cells recognize antigenic peptides contained within DRB1*1301 that are expressed in liver antigen presenting cells [18]. After activation, CD4+ cells become effector Th1 cells, which then secrete IFN-gamma [18]. IFN-gamma enhances the expression of HLA class molecules on hepatocytes, which become the targets of the CD8+ cells/NK cells [18]. Therefore, in pediatric $\mathrm{AIH}$, there is an increase in the frequency of KIR2DS4-Full Length gene that alters the KIRs, which in combination with HLA-DRB1*1310 has a strong synergistic effect to activate liver $\mathrm{T}$ cells and produce inflammation [18]. This suggests a genetic influence for the early onset of type $1 \mathrm{AIH}$ [18]. The patient in this case was also diagnosed with early onset AIH type 1. Perhaps, a similar mechanism of upregulation and over-activation of a particular subclass of $\mathrm{T}$ cells in the liver and bowel are the source of inflammation. As previously discussed, repeated low-grade bacterial infections, and the circulation of pro-inflammatory bacterial peptides or abnormal secondary bile acids from the bowel to the liver via enterohepatic circulation cause fibrosis and hepatobiliary disease in PSC [6, 7]. Lymphocytes are activated to recognize the bowel and hepatic endothelium with memory $T$ cells that induce the inflammation [6]. Therefore, just like VEO-IBD and early onset AIH, PSC has a connection with the interactions of the microbiota and the T cells.

The patient also had a strong family history of IBD on the maternal side. Perhaps, the fecal transplant should not have come from the mother although she was healthy. The prolonged fever after the fecal transplant helped promote an earlier diagnosis of her autoimmune diseases. The child may have benefitted more from a stool transplant from a stool bank. Currently, fecal transplant is seen as a cost-effective, FDA-approved, safe, and accepted treatment for severe Clostridioides difficile refractory to antibiotics [19]. There are emerging data involving the use of fecal transplant in managing IBD, autoimmune disorders, obesity, diabetes, and numerous non-GI disorders [12, 15, 19-22]. Fecal transplant has been studied for the treatment of both Ulcerative Colitis and Crohn's disease [12]. After fecal transplant, the microbiota can enter the mesenteric lymph node, leading to an interaction between

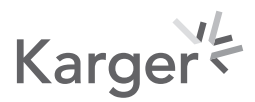


regulatory T cells and the microbiota [23]. This clinical case in our young patient also demonstrates that careful selection of the donor is important. What is unknown is the exact immune system interaction of the recipient to the stool from a donor. In the future, we may be able to map out the microbiota of the donor in order to match it better with the recipients and the type of autoimmune disease that is being treated.

This case is unique as it shows the time line of how immune dysregulation evolve over the years to complex autoimmune disease. Diagnosis can be difficult and confusing in this age because the disease can mimic other diseases. In this clinical case, it took 6 years until the child's pathology, clinical presentation, endoscopic findings, and labs were transpicuous. Based on the first histologic findings of eosinophilia from the endoscopy, the diagnosis was incorrectly thought to be allergic enterocolitis. Recent articles have now reported eosinophils the mucosa of patients with VEO-IBD, which can create confusion with pathology diagnosis, and delay appropriate medical therapy. Patients with VEO-IBD can have monogenic mutations that should be evaluated by a geneticist and immunologist. The possible link among VEO-IBD, AIH, and PSC is the microbial dysbiosis and the interactions with T cells that produce inflammation. Clinicians need to be aware of cases like this so that the differential for VEO-IBD should be considered early and the patients should be followed very closely.

\section{Acknowledgements}

This manuscript does not include any nonauthor contributors to acknowledge.

\section{Statement of Ethics}

Written informed consent was obtained by the parents for the patient who is under 18 years in order to publish this manuscript. This study protocol was reviewed, and the need for approval was waived by our institution's IRB at Johns Hopkins All Children's Hospital.

\section{Conflict of Interest Statement}

The authors have no conflicts of interest to declare.

\section{Funding Sources}

No funding was obtained for this case report.

\section{Author Contributions}

Dr. Aditi Dey, Jacquelin Peck, Dr. Michael Wilsey, Dr. Jolan Walter, and Dr. Sara Karjoo contributed a significant portion of drafting the manuscript and approved the final draft submitted. Dr. Aditi Dey obtained patient data and contributed greatly to the final manuscript. Jacquelin Peck contributed greatly to the writing and editing of manuscript. Dr. Michael Wilsey provided expertise on the field and contributed to the manuscript. Dr. Jolan Walter provided expertise on the field and contributed to the writing and editing of the manuscript. Dr. Sara Karjoo provided supervision, expertise on the topic, idea creation, and writing and

\section{Karger'"}


Dey et al.: Young Girl with VEO-IBD, AIH, and PSC

editing of the manuscript. All authors read and approved the final manuscript. Dr. Sara Karjoo is the article guarantor.

\section{Data Availability Statement}

The case data are from medical records on the Johns Hopkins All Children's Hospital electronic medical record system.

\section{References}

1 Shim JO. Recent advance in very early onset inflammatory bowel disease. Pediatr Gastroenterol Hepatol Nutr. 2019 Jan;22(1):41-9.

2 Crowley E, Muise A. Inflammatory bowel disease: what very early onset disease teaches us. Gastroenterol Clin North Am. 2018 Dec;47(4):755-72.

3 Kelsen JR, Russo P, Sullivan KE. Early-onset inflammatory bowel disease. Immunol Allergy Clin North Am. 2019 Feb;39(1):63-79.

4 Kerkar N, Mack CL. Autoimmune hepatitis. In: Suchy F, editor. Liver disease in children. Cambridge: Cambridge University Press; 2014. p. 311-21.

5 Damman JL, Rodriguez EA, Ali AH, Buness CW, Cox KL, Carey EJ, et al. Review article: the evidence that vancomycin is a therapeutic option for primary sclerosing cholangitis. Aliment Pharmacol Ther. 2018 Apr;47(7): 886-95.

6 Miethke AG, Balistreri WF. Sclerosing. In: Suchy F, editor. Liver disease in children. Cambridge: Cambridge University Press; 2014. p. 322-40.

7 Pathak S, Kamat D. Autoimmune hepatitis in children. Pediatr Ann. 2018 Feb;47(2):e81-6.

8 Woodhouse CA, Patel VC, Singanayagam A, Shawcross DL. Review article: the gut microbiome as a therapeutic target in the pathogenesis and treatment of chronic liver disease. Aliment Pharmacol Ther. 2018 Jan;47(2): 192-202.

9 Conrad MA, Carreon CK, Dawany N, Russo P, Kelsen JR. Distinct histopathological features at diagnosis of very early onset inflammatory bowel disease. J Crohns Colitis. 2019 Apr;13(5):615-25.

10 Uhlig HH, Schwerd T, Koletzko S, Shah N, Kammermeier J, Elkadri A, et al. The diagnostic approach to monogenic very early onset inflammatory bowel disease. Gastroenterology. 2014 Nov;147(5):990-1007.e3.

11 Conrad MA, Kelsen JR. Genomic and immunologic drivers of very early-onset inflammatory bowel disease. Pediatr Dev Pathol. 2019 May-Jun;22(3):183-93.

12 Tan P, Li X, Shen J, Feng Q. Fecal microbiota transplantation for the treatment of inflammatory bowel disease: an update. Front Pharmacol. 2020 Sep;11:574533.

13 Jeyaratnam J, Frenkel J. Management of mevalonate kinase deficiency: a pediatric perspective. Front Immunol. 2020 Jun;11:1150.

14 Sullivan KE, Conrad M, Kelsen JR. Very early-onset inflammatory bowel disease: an integrated approach. Curr Opin Allergy Clin Immunol. 2018 Dec;18(6):459-69.

15 Teich N, Weber M, Stallmach A. First occurrence of severe extraintestinal manifestations of Crohn's disease following faecal microbiota transplantation. J Crohns Colitis. 2016 Oct;10(10):1254-5.

16 Shi Y, Dong Y, Huang W, Zhu D, Mao H, Su P. Fecal microbiota transplantation for ulcerative colitis: a systematic review and meta-analysis. PLoS One. 2016 Jun;11(6):e0157259.

17 Uygun A, Ozturk K, Demirci H, Oger C, Avci IY, Turker T, et al. Fecal microbiota transplantation is a rescue treatment modality for refractory ulcerative colitis. Medicine. 2017 Apr;96(16):e6479.

18 Podhorzer A, Paladino N, Cuarterolo ML, Fainboim HA, Paz S, Theiler G, et al. The early onset of type 1 autoimmune hepatitis has a strong genetic influence: role of HLA and KIR genes. Genes Immun. 2016 Apr;17(3): $187-92$.

19 Lee WJ, Lattimer LDN, Stephen S, Borum ML, Doman DB. Fecal microbiota transplantation: a review of emerging indications beyond relapsing clostridium difficile toxin colitis. Gastroenterol Hepatol. 2015 Jan;11(1):24-32.

20 Li Y, Tang R, Leung PSC, Gershwin ME, Ma X. Bile acids and intestinal microbiota in autoimmune cholestatic liver diseases. Autoimmun Rev. 2017 Sep;16(9):885-96.

21 Vrieze A, de Groot PF, Kootte RS, Knaapen M, van Nood E, Nieuwdorp M. Fecal transplant: a safe and sustainable clinical therapy for restoring intestinal microbial balance in human disease? Best Pract Res Clin Gastroenterol. 2013 Feb;27(1):127-37.

22 Xu MQ, Cao HL, Wang WQ, Wang S, Cao XC, Yan F, et al. Fecal microbiota transplantation broadening its application beyond intestinal disorders. World J Gastroenterol. 2015 Jan;21(1):102-11.

23 Fischer M, Kao D, Mehta SR, Martin T, Dimitry J, Keshteli AH, et al. Predictors of early failure after fecal microbiota transplantation for the therapy of clostridium difficile infection: a multicenter study. Am J Gastroenterol. 2016 Jul;111(7):1024-31. 